\title{
Angiotensin II-dependent Src and Smad1 signaling pathway is crucial for the development of diabetic nephropathy
}

\author{
Akira Mima ${ }^{1}$, Takeshi Matsubara ${ }^{1}$, Hidenori Arai ${ }^{2}$, Hideharu Abe $^{3}$, Kojiro Nagai ${ }^{1}$, \\ Hiroshi Kanamori ${ }^{1}$, Eriko Sumi ${ }^{1}$, Toshikazu Takahashi ${ }^{3}$, Noriyuki Iehara ${ }^{1}$, \\ Atsushi Fukatsu ${ }^{1}$, Toru Kita ${ }^{4}$ and Toshio Doi $^{3}$ \\ ${ }^{1}$ Department of Nephrology, Kyoto University Graduate School of Medicine, Kyoto, Japan; ${ }^{2}$ Department of \\ Geriatric Medicine, Kyoto University Graduate School of Medicine, Kyoto, Japan; ${ }^{3}$ Department of Clinical \\ Biology and Medicine, Tokushima University School of Medicine, Tokushima, Japan and ${ }^{4}$ Department of \\ Cardiovascular Medicine, Kyoto University Graduate School of Medicine, Kyoto, Japan
}

\begin{abstract}
Angiotensin II (Ang II) is known to play a pivotal role in the development of diabetic nephropathy. However, the precise mechanism of Ang II-mediated effects on diabetic nephropathy is still unknown. We have reported that Smad1 plays a key role in diabetic mesangial matrix expansion and directly regulates the transcription of type IV collagen (Col4) in vitro and in vivo. Here we examined the effect of Ang II on the expression of Smad1 and mesangial matrix expansion in streptozotocin (STZ)-induced diabetic rats in vivo, using Ang II type 1 receptor blocker, olmesartan. We also examined the signaling mechanism by which Ang II induces mesangial matrix expansion in vitro. Treatment of diabetic rats with low-dose olmesartan for 20 weeks reduced albuminuria and hyperfiltration without affecting blood pressure and inhibited mesangial matrix expansive changes and the expression of Col4 and smooth muscle alpha actin compared with those in untreated rats. Immunohistochemical staining and Western blotting showed that the increased expression of Smad1, phospho-Smad1, and phospho-Src was inhibited by olmesartan. Ang II induced Col4 synthesis and increased expression of phosphoSrc and phospho-Smad1 in cultured mesangial cells, which was blocked by olmesartan. PP2, a Src tyrosine kinase inhibitor, and overexpression of dominant negative Src also reduced the phosphorylation of Smad1. Moreover, addition of small-interfering RNA against Src significantly reduced the phosphorylation of Smad1 and synthesis of Col4. Taken together, these results indicate that Ang II can regulate the development of mesangial matrix expansion in the early phase of diabetic nephropathy through Src and Smad1.

Laboratory Investigation (2006) 86, 927-939. doi:10.1038/labinvest.3700445; published online 12 June 2006
\end{abstract}

Keywords: angiotensin II; diabetic nephropathy; mesangial matrix expansion; Smad1; Src; type IV collagen

Diabetic nephropathy is the most common cause of end-stage renal disease and a major contributing cause of morbidity and mortality in patients with diabetes in many countries. This disease is characterized by thickening of glomerular basement membrane and mesangial matrix expansion. Moreover, it is well known that mesangial matrix expansion is generally accompanied by various tubulointerstitial damages followed by nephron loss to end-stage renal failure. ${ }^{1-3}$ The decline of glomerular filtration rate is associated with the increasing number of obsolescent glomeruli and the severity of

Correspondence: Dr H Arai, MD, PhD, Department of Geriatric Medicine, Kyoto University Graduate School of Medicine, 54 Kawahara-cho, Shogoin, Sakyo-ku, Kyoto 606-8507, Japan.

E-mail: harai@kuhp.kyoto-u.ac.jp

Received 27 April 2006; revised 9 May 2006; accepted 11 May 2006; published online 12 June 2006 mesangial matrix expansion in type I diabetes. ${ }^{4}$ The early stage of diabetic nephropathy is associated with glomerular hyperfiltration and glomerular hypertrophy, but not collapse of glomerular capillaries. Therefore, mesangial matrix expansion is one of the most important pathological findings in the early phase of diabetic nephropathy. Interruption of the renin-angiotensin system (RAS) using angiotensin II (Ang II) type 1 receptor blocker (AT1 antagonist) or an angiotensin-converting enzyme inhibitor has been proved clinically effective in slowing the decline in renal function of several nephropathies, including diabetic nephropathy ${ }^{5-8}$ and slows the progression of renal disease and delays the need for dialysis or transplantation in diabetic patients. ${ }^{9}$ Several reports indicate that RAS is accelerated in early diabetic nephropathy ${ }^{10}$ and that intrarenal Ang II expression is already augmented before apparent diabetic nephropathy. ${ }^{11}$ 
Ang II can increase vascular tone by inducing contraction of vascular smooth muscle and mesangial cells and promote cellular proliferation and extracellular matrix (ECM) synthesis through direct effects, both hemodynamic and nonhemodynamic, or via induction of growth factors. ${ }^{12-14}$ These data support the notion that Ang II can worsen diabetic nephropathy. However, little is known about the precise molecular mechanisms by which Ang II exerts its effects on diabetic nephropathy.

It is well known that mesangial matrix expansion is characterized by increased amounts of ECM, including type IV collagen (Col4), laminin, type I and III collagens, heparan sulfate proteoglycan, and fibronectin. ${ }^{15-17}$ Striker et $a l^{16}$ show that various peptide growth factors mediate the regulation of Col4, which is the key component is in the pathophysiology of diabetic nephropathy. We have recently reported that Smad1 is a key molecule for direct transcriptional regulation of $\mathrm{Col} 4$ in diabetic nephropathy, ${ }^{18}$ and that the glomerular expression of Smad1 is significantly increased in diabetic rats with mesangial matrix expansion. ${ }^{19}$ However, the effect of Ang II on Smad1 in the mesangial matrix expansion of diabetic nephropathy is not fully elucidated.

Src is a ubiquitously expressed nonreceptor protein-tyrosine kinase ${ }^{20}$ and is involved in signaling by several cytokines and growth factors such as Ang $\mathrm{II}^{21}$ and platelet-derived growth factor (PDGF). ${ }^{22}$ It is well known that Src may contribute to both cell proliferation and migration. ${ }^{23,24}$ PDGF plays a role as a mesangial cell mitogen and causes proliferative glomerulopathy. ${ }^{25,26}$ Our previous data also show that the development of glomerulosclerosis from mesangial proliferation is dependent on PDGF, signal transducer and activator of transcription 3, and Smad1 pathway. ${ }^{27}$ However, it is not known whether Ang II activates Smad1 or what is the signaling mechanism involved in Ang II-mediated Smad1 activation in mesangial cells. In the present study, therefore, we examined whether Ang II can modulate Smad1-mediated signaling involved in mesangial matrix expansion in diabetic nephropathy in vivo and in vitro. In vivo, we used streptozotocin (STZ)-induced rats receiving low-dose AT1 antagonists and examined the role of Ang II in Smad1-mediated mesangial matrix expansion. In vitro, by using primary culture mesangial cells, the effect of Ang II on Smad1 and Src, and the role of Src on Smad1-mediated signaling in mesangial cells were examined.

\section{Materials and methods}

\section{Animals}

Male Sprague-Dawley rats weighing 170-200 g were used in study. Rats were purchased from Shimizu Laboratory Animal center (Hamamatsu, Japan). The animals were housed under specific pathogen- free conditions at the Animal Facility of Kyoto University, Faculty of Medicine. All the animal experiments were performed in accordance with institutional guidelines, and the Review Board of Kyoto University granted ethical permission to this study.

\section{Induction of STZ Diabetic Rat}

Male rats weighing 170-200 g were made diabetic by a single intravenous injection of STZ (Wako, Osaka, Japan) (55 mg/kg body weight) in $0.05 \mathrm{~mol} / \mathrm{l}$ citrate buffer ( $\mathrm{pH}$ 4.5). Rats receiving an injection of citrate buffer were used as controls. The levels of blood glucose were determined 2 days after injection of STZ or vehicle, and rats with blood glucose levels more than $16.7 \mathrm{mmol} / \mathrm{l}$ were used as diabetic. ${ }^{28}$

\section{Protocol of Treatment with Ang II Type 1 Receptor Blocker in STZ Rats}

The rats were divided into four groups for the investigation: group 1, control rats treated with vehicle; group 2, control rats treated with olmesartan (Sankyo Co., Tokyo, Japan) (0.6 mg/kg/day); group 3, STZ-diabetic rats treated with vehicle; group 4, STZ-diabetic rats treated with olmesartan $(0.6 \mathrm{mg} / \mathrm{kg} /$ day $)$. The dosage of olmesartan was determined based on the previous reports. ${ }^{29}$ Olmesartan or vehicle was administered 4 weeks after the injection of STZ or vehicle. At 24 weeks after STZ injection, the rats were weighted and killed. Blood was collected at the time of killing. Serum creatinine and blood urea nitrogen were measured with a Hitachi Mode 736 autoanalyzer (Hitachi, Tokyo, Japan). ${ }^{30}$ The levels of blood glucose and HbA1c were measured using One touch ultra (Johnson \& Johnson, Tokyo, Japan) and DCA2000 analyzer (Bayer Medical, Tokyo, Japan). Before killing, creatinine and albumin were measured from 24-h urine collection.

\section{Histological Examination}

\section{Light microscopy}

After removal of the kidney, tissue blocks for light microscopy examination were fixed in methyl Carnoy's solution and embedded in paraffin. Sections $(2 \mu \mathrm{m})$ were stained with periodic acid-Schiff methenamine (PASM).

\section{Immunohistochemistry}

Kidney sections were processed for immunohistochemistry following standard procedures. To study Col4 and Smad1, ethyl Carnoy's solution-fixed and paraffin-embedded tissue blocks were used. Kidney sections were rehydrated and treated with $0.3 \%$ hydrogen peroxide in methanol for $30 \mathrm{~min}$. To eliminate nonspecific staining, sections were incubated with the appropriate preimmune serum 
for $60 \mathrm{~min}$ at room temperature, and then incubated with AvidinD and Biotin blocking solutions (Vector, Burlingham, CA, USA) for $15 \mathrm{~min}$ each. Sections were incubated with anti-Col4 antibody (1:200 dilution) (PROGEN BIOTECHNIK GMBH, Heidelberg, Germany) or anti-Smad1 antibody (1:100 dilution) (Santa Cruz Biotechnology, CA, USA) overnight at $4^{\circ} \mathrm{C}$, and then incubated with the appropriate biotinylated secondary antibodies followed by incubation with the avidin-biotin peroxidase complex (Vectastain Elite ABC kit, Vector). Peroxidase conjugates were subsequently localized using diaminobenzidine tetrahydrochloride. To examine the expression of smooth muscle alpha actin $(\alpha$-SMA), the tissues were snap-frozen in cold acetate in OCT compound (Sakura Finetechnical, Tokyo, Japan), and then cut in 4- $\mu$ m-thick sections and fixed in acetone for $10 \mathrm{~min}$, and treated with $0.3 \%$ hydrogen peroxide in methanol for $30 \mathrm{~min}$. Sections were treated in the same manner as Col4 and Smad1 with anti- $\alpha$-SMA antibody (1A4 at 1:100 dilution) (Sigma, St Louis, MO, USA).

\section{Quantitation of light microscopy}

Glomerular morphometry was evaluated in PASMstained tissues. The glomerular surface area and the PASM-positive area/glomerular area (\%) were measured using an image analyzer with microscopy (IPAP, Image Processor for Analytical Pathology; Sumitomo Chemical Co., Osaka, Japan) as described $^{31}$ For each animal, 50 glomeruli were analyzed.

\section{Immunofluolescence staining and quantitation of} glomerular Col4 expression

Immunofluorescence staining for Col4, pSmad1, and pSrc was performed using frozen kidney sections. Sections $(4 \mu \mathrm{m})$ were fixed in acetone, blocked with $10 \%$ donkey serum, and incubated overnight with anti-Col4 antibody (1:200 dilution) (PROGEN), anti-phospho Smad1/5/8 antibody (1:100 dilution) (Cell Signaling Technology, Beverly, MA, USA), or anti-phospho Src (Cell Signaling) (1:50 dilution).

To quantificate the expression of Col4, the Col4positive area in glomeruli was measured using Image-Pro PLUS (Media Cybernetics, silverspring, MD, USA) as described. ${ }^{32}$ For each animal, 50 glomeruli were evaluated.

\section{Isolation of Glomeruli}

Rat glomeruli were isolated from renal cortex of rats using the differential sieving method. The purity of the glomeruli was $>90 \% .^{33}$

\section{Cell Culture}

We previously reported that cultured rat mesangial cells changed their phenotypes during multiple passages. $^{34}$ Therefore, we freshly isolated mouse mesangial cells from normal 4-week-old mice $(\mathrm{C} 57 \mathrm{BL} / 6 \mathrm{~J} \times \mathrm{SJL} / \mathrm{J})$ and identified according to the method previously described. ${ }^{35}$ The mesangial cells were maintained in B medium (a 3:1 mixture of minimal essential medium/F12 modified with trace elements) (Nissui Pharmaceutical Co., Ltd., Tokyo, Japan) supplemented with $1 \mathrm{mM}$ glutamine, penicillin at $100 \mathrm{U} / \mathrm{ml}$, streptomycin at $100 \mu \mathrm{g} / \mathrm{ml}$ (Invitrogen, Carisbad, CA, USA), and $20 \%$ fetal calf serum (FCS) (Invitrogen). The cultured cells fulfilled the criteria generally accepted for glomerular mesangial cells. These cells were plated on 100-mm plastic dishes (Nalge Nunc International, Roskilde, Denmark) and maintained in B medium $/ 20 \%$ FCS. After $48 \mathrm{~h}$ incubation, cells were starved in DMEM containing $0.5 \%$ FCS before treatment. Stimulation with Ang II (Sigma), olmesartan (Sankyo), PP2 (Calbiochem, Darmstadt, Germany), or U0126 (Calbiochem) was carried out in DMEM containing 0.5\% FCS at $37^{\circ} \mathrm{C}$ for the indicated times.

\section{Cell Transfection}

Mesangial cells $\left(1.0 \times 10^{5}\right)$ were seeded into six-well plates (Nunc). After $6 \mathrm{~h}$, the cells were transfected with $2 \mu \mathrm{g}$ of expression vector encoding dominant negative Src (Upstate Biotechnology, Charlottesville, VA, USA) or with $2 \mu \mathrm{g}$ of mock vector (pUSEamp(-); Upstate) using Fugene6 transfection reagent (Roche, Penzberg, Germany) according to the manufacturer's instructions with some modifications. After $24 \mathrm{~h}$ of transfection, cells were starved in DMEM containing $0.5 \%$ FCS before treatment. After $48 \mathrm{~h}$ incubation, cells were stimulated with or without Ang II (Sigma). The cells were harvested in lysis buffer after stimulation and Western blotting was performed as reported below.

\section{Small-Interfering RNA}

Mesangial cells $\left(1.0 \times 10^{5}\right)$ were seeded into six-well plates (Nunc) and were grown until $60-80 \%$ confluent. The c-Src small-interfering RNA (si RNA) (SMARTpool; Dharmacon, Lafayette, CO, USA) or control siRNA (SMARTpool; Dharmacon) was combined with DharmaFECT transfection reagent (Dharmacon), and the cells were transfected according to the recommended protocol with siRNA $(100 \mathrm{nM}$ final concentration). After $48 \mathrm{~h}$ of transfection, cells were starved in DMEM containing 0.5\% FCS before treatment. After $48 \mathrm{~h}$ incubation, cells were stimulated with or without Ang II. The cell lysates were then prepared for Western blot analysis.

\section{Western Blotting}

Isolated glomeruli or harvested mesangial cells were suspended in RIPA buffer $(50 \mathrm{mM}$ Tris, $\mathrm{pH} 7.5$, $150 \mathrm{mM} \mathrm{NaCl}, 1 \%$ Nonindet P-40, 0.25\% SDS, $1 \mathrm{mM}$ $\mathrm{Na}_{3} \mathrm{VO}_{4}, 2 \mathrm{mM}$ EDTA, $1 \mathrm{mM}$ phenylmethylsulfonyl fluoride, $10 \mu \mathrm{g} / \mathrm{ml}$ of aprotinin), and incubated for 
$1 \mathrm{~h}$ at $4^{\circ} \mathrm{C}$. After centrifugation, the supernatants were used as total cell lysates. In all, $30 \mu \mathrm{g}$ of each sample was applied to SDS-PAGE. After electrophoresis, the proteins were transferred to nitrocellulose filters (Schleicher \& Schuell, Keene, NH, USA). The blots were subsequently incubated with anti-Smad1 (Upstate), anti-phospho Smad1/5/8 (Cell Signaling), anti-Col4 antibody (PROGEN) (Biodesign, Saco, ME, USA), anti-nonphospho Src (Cell Signaling), anti-phospho Src (Cell Signaling), antip44/42 mitogen-activated protein (MAP) kinase antibody (Oncogene, San Diego, CA, USA), antiphospho p44/42 MAP kinase antibody (Cell Signaling), anti- $\beta$-actin antibody (Cell Signaling), or anti- $\alpha$ SMA antibody (Sigma), following by incubation with horseradish peroxidase-conjugated goat antirabbit IgG, and sheep anti-mouse IgG (Amersham Biosciences, Piscataway, NJ, USA). The immunoreactive bands were visualized using horseradish peroxidase-conjugated secondary antibody and the enhanced chemiluminescent system (Amersham Biosciences). These bands were quantificated by imaging densitometer, Science Lab 99 Image Gauge (Fujifilm, Tokyo, Japan).

\section{Glomerular Filtration Rate and the Measurement of Urinary Albumin Excretion}

Urine volume $(\mathrm{Vu})$ was measured at 24 weeks by $24 \mathrm{~h}$ urine collection from rats housed in individual metabolic cages. During the urine collection, the rats were allowed free access to food and water. Serum and urine creatinine concentrations $(\mathrm{Cp}$ and $\mathrm{Cu}$ ) were measured, and glomerular filtration rate (GFR) was calculated by the following equation: $\mathrm{GFR}=(\mathrm{Cu} / \mathrm{Cp}) \times \mathrm{Vu} /$ body weight. $^{36}$ The albumin concentration in the urine was measured by Nephrat (Exocell Inc., Philadelphia, PA, USA).

\section{Statistical Analysis}

The data are expressed as the mean \pm the standard deviation (s.d.). Comparison among more than two groups was performed by one-way analysis of variance (ANOVA) followed by the post hoc analysis (Bonferroni/Dunn test) to evaluate statistical significance between the two groups. All analyses were performed using StatView (SAS Institute, Cary, CA, USA). Statistical significance was defined as $P<0.05$.

\section{Results}

\section{Characteristics of Four Groups}

Table 1 shows the characteristics of four groups of rats at 24 weeks after STZ injection. Blood sugar (BS) and HbA1c were increased, and the body weight (WT) was decreased in both diabetic groups compared with those in nondiabetic groups. There was no significant difference in systolic blood pressure (BP) between the four groups.

\section{AT1 Antagonist Olmesartan Ameliorates the Renal Function of STZ Rats}

Figure 1 shows the creatinine clearance and urinary albumin excretion of four groups of rats determined at the end of the experiment. The diabetic rats treated with vehicle (group 3) showed increased creatinine clearance and urinary albumin excretion compared with control rats (group 1 and group 2). However, the diabetic rats treated with olmesaran (group 4) showed less creatinine clearance and urinary albumin excretion than untreated diabetic rats. The glomerular hypertrophy was recognized in untreated diabetic rats, whereas olmesartan ameliorated it. In treated control rats, olmesartan also ameliorated hyperfiltration, albuminuria, and glomerular hypertrophy owing to aging.

\section{Olmesartan Inhibits Mesangial Matrix Expansion in STZ Rats}

To examine the effect of olmesartan on diabetic mesangial matrix expansion, we assessed mesangial matrix expansion by measuring PASM-positive areas in the four groups of rats. Untreated diabetic rats showed increased degree of mesangial matrix area and mesangial matrix expansion compared with control rats. When we treated diabetic rats with olmesartan for 20 weeks, both mesangial matrix area and mesangial matrix expansion were significantly ameliorated (Figure 2A). Figure 2B shows a representative light microscopic picture in each group. To investigate the effect of olmesartan on the morphological changes seen diabetic mesangial matrix expansion, such as increased Col4 and $\alpha$-SMA expression, we examined their

Table 1 Physiological characteristics of control and diabetic rats with or without olmesartan treatment

\begin{tabular}{|c|c|c|c|c|c|}
\hline & Number & Body WT (g) & $H b A 1 c(\%)$ & $B S(m g / d l)$ & Systolic BP (mmHg) \\
\hline Control & 6 & $627 \pm 10$ & $2.7 \pm 0.3$ & $114 \pm 12$ & $113 \pm 8$ \\
\hline Control+Olm & 6 & $631 \pm 12$ & $2.6 \pm 0.1$ & $119 \pm 11$ & $112 \pm 3$ \\
\hline Diabetes & 7 & $320 \pm 75^{*}$ & $7.0 \pm 1.1^{*}$ & $382 \pm 97$ * & $112 \pm 2$ \\
\hline Diabetes+Olm & 7 & $352 \pm 53 *$ & $6.4 \pm 0.9^{*}$ & $459 \pm 64^{*}$ & $111 \pm 4$ \\
\hline
\end{tabular}

The rats were separated into control and diabetic groups with or without olmesartan treatment. Rats with olmesartan treatment (Olm) were administered $0.6 \mathrm{mg} / \mathrm{kg}$ olmesartan. After 24 weeks of STZ injection, systolic blood pressure (Systolic BP) was measured by the cuff-tailed method, and the rats were weighed (Body WT) and killed. Blood was taken to evaluate blood sugar (BS) and HbA1c. The data are expressed as the means \pm s.d. ${ }^{*} P<0.05$ vs control rats. 

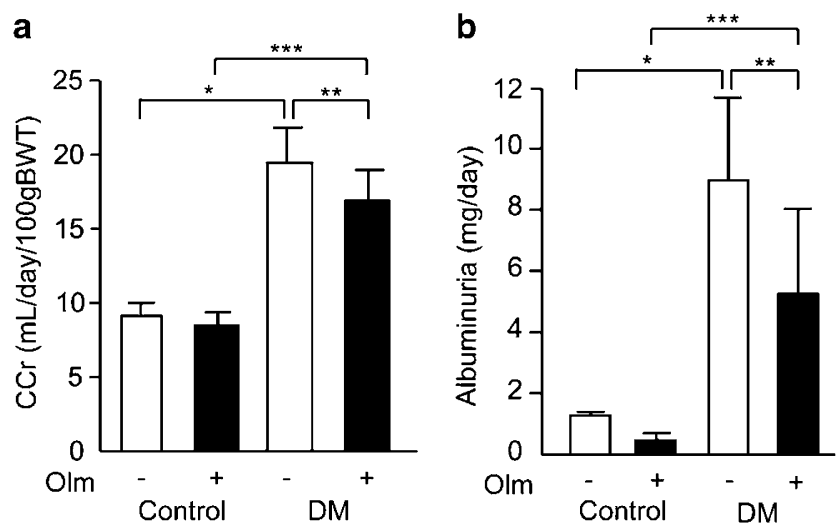

C

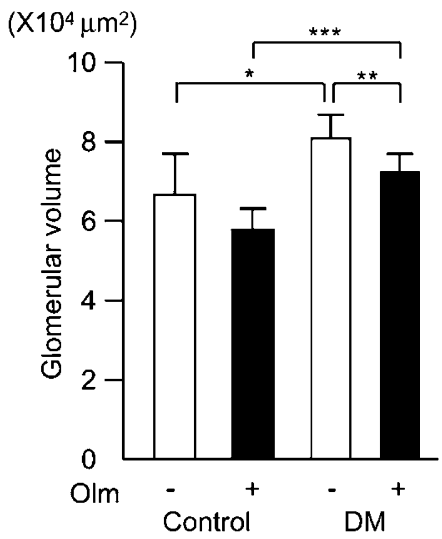

Figure 1 Effects of olmesartan on creatinine clearance and albuminuria in STZ rats. (a) Creatinine clearance (Ccr) was calculated and normalized as described in Materials and methods. (b) Albuminuria was measured by Nephrat. The data are expressed as the means \pm s.d. ( $n=6$ in the control group and $n=7$ in the diabetic group). (c) Glomerular volume was detected by IPAP as described in Materials and methods. ${ }^{*} P<0.05$ vs control rats treated with vehicle (group 1 ). ${ }^{* *} P<0.05$ vs diabetic rats treated with vehicle (group 3 ). ${ }^{* * *} P<0.05$ vs control rats treated with olmesartan (group 2).

expression in the four groups. In untreated diabetic rats, the glomerular expression of Col4 was significantly increased compared with that in control rats and their expression was mostly localized at the mesangial area by immunohistochemical analysis. Olmesartan treatement inhibited the glomerular expression of Col4 by approximately $30 \%$ compared with untreated diabetic rats (Figure $3 \mathrm{C}$ ). We also examined the glomerular expression of $\alpha$-SMA by Western blotting and found that $\alpha$-SMA was almost undetectable in nondiabetic rats. However, the expression of $\alpha$-SMA was significantly increased in untreated diabetic rats, compared with control rats. The expression was also decreased by olmesartan treatment (Figure 3A). Figure 3D shows a representative light microscopic picture in each group. In untreated control rats, slight mesangial matrix expansion due to aging was recognized and olmesartan ameliorated this matrix expansion. The decreased glomerular surface area as a denominator of mesangial matrix fraction in treated control rats led that they were larger than untreated control rats (Figure 2A). The same phenomenon was recognized in Col4-positive staining area (Figure 3C).

Glomerular Expression of Smad1, Phospho-Smad1 (pSmad1), Src, and Phospho-Src (pSrc) is Decreased by Olmesartan

We next examined the glomerular expression of Smad1 in these rats. By Western blotting and immunohistochemistry, Smad1 expression was remarkably increased in untreated diabetic rats compared with nondiabetic rats. However, its expression was decreased by olmesartan treatment (Figure 4). We also examined whether the phosphorylation and translocation of Smad1 were affected by olmesartan treatment in diabetic rats. By Western blotting and immunohistochemistry, pSmad1 was hardly detected in nondiabetic rats, whereas pSmad1 was markedly increased and its expression was localized in the nucleus in untreated diabetic rats. As shown in the Smad1 expression, the glomerular expression of pSmad1 was significantly decreased by olmesartan treatment (Figure 4). To investigate the role of $\mathrm{Src}$ in mesangial matrix expansion in diabetic nephropathy, we examined the glomerular expression of Src along with phosphorylated Src by Western blotting. The glomerular expression of Src and pSrc was markedly increased, compared with control rats. The increased expression was also decreased by olmesartn treatment as in Smad1 and pSmad1 (Figure 5A and B). By immunohistochemistry, we also found that increased pSrc was localized at the mesangial area. As shown in the Smad1 and pSmad1 expression, the glomerular expression of pSrc was significantly reduced by olmesartan treatment (Figure 5C).

\section{In Vitro Effect of Olmesartan, PP2, and U0126 on Ang II-Mediated Signaling in Mesangial Cells}

To determine whether Ang II can activate Smad1 and increase the synthesis of Col4, and whether this effect can be blocked by olmesartan, cultured mesangial cells were incubated with $0.1 \mu \mathrm{M}$ Ang II for of the indicated times or with various concentrations of Ang II for $15 \mathrm{~min}$. As shown in Figure 6a, Ang II caused the phosphorylation of Smad1 in a time-dependent manner, peaking at $15 \mathrm{~min}$. Stimulation of mesangial cells with various concentrations of Ang II showed a dose-dependent increase in $\mathrm{pSmad} 1$ reaching a plateau at $1 \mu \mathrm{M}$ (Figure 6b). Ang II also stimulated the phosphorylation of Src and the synthesis of Col4 (Figure 6c). Next, we examined whether olmesartan can prevent the Ang II-induced phosphorylation of Smad1, Src, and the synthesis of Col4. As shown in Figure 6c, olmesartan inhibited the phosphorylation of Smad1 and Src and the expression of Col4 after Ang II stimulation. To show the interaction between Src and Smad1, we examined whether PP2 $(10 \mu \mathrm{M})$, an Src tyrosine kinase 
A

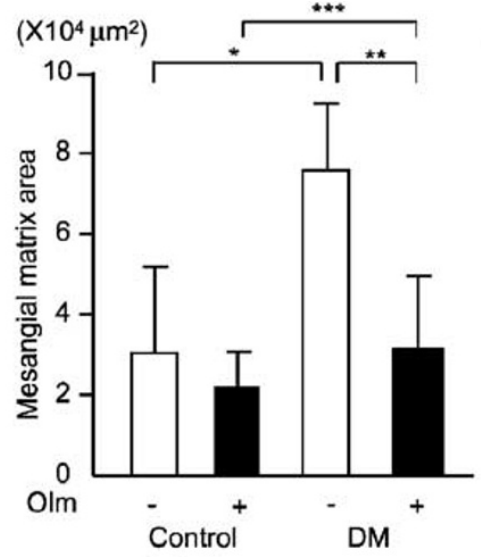

B
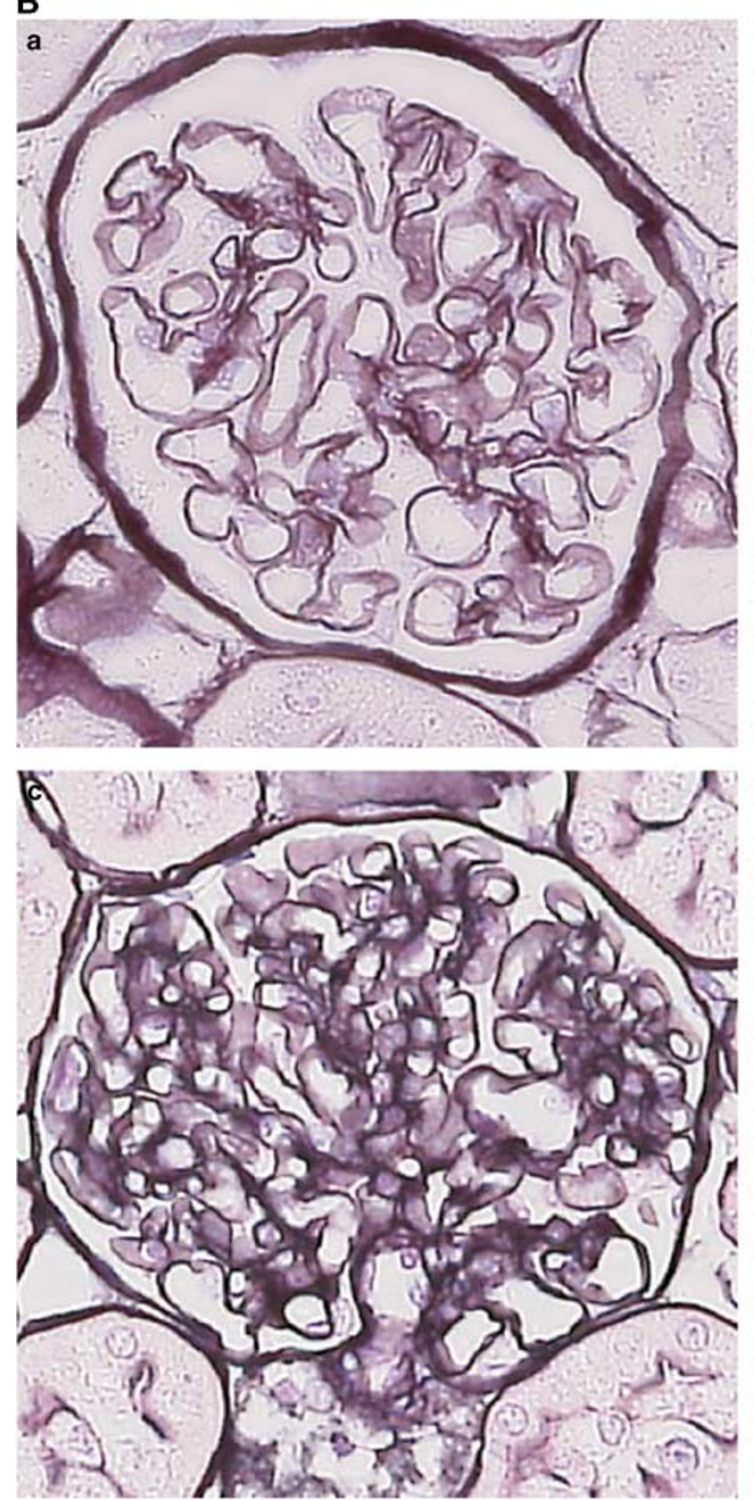
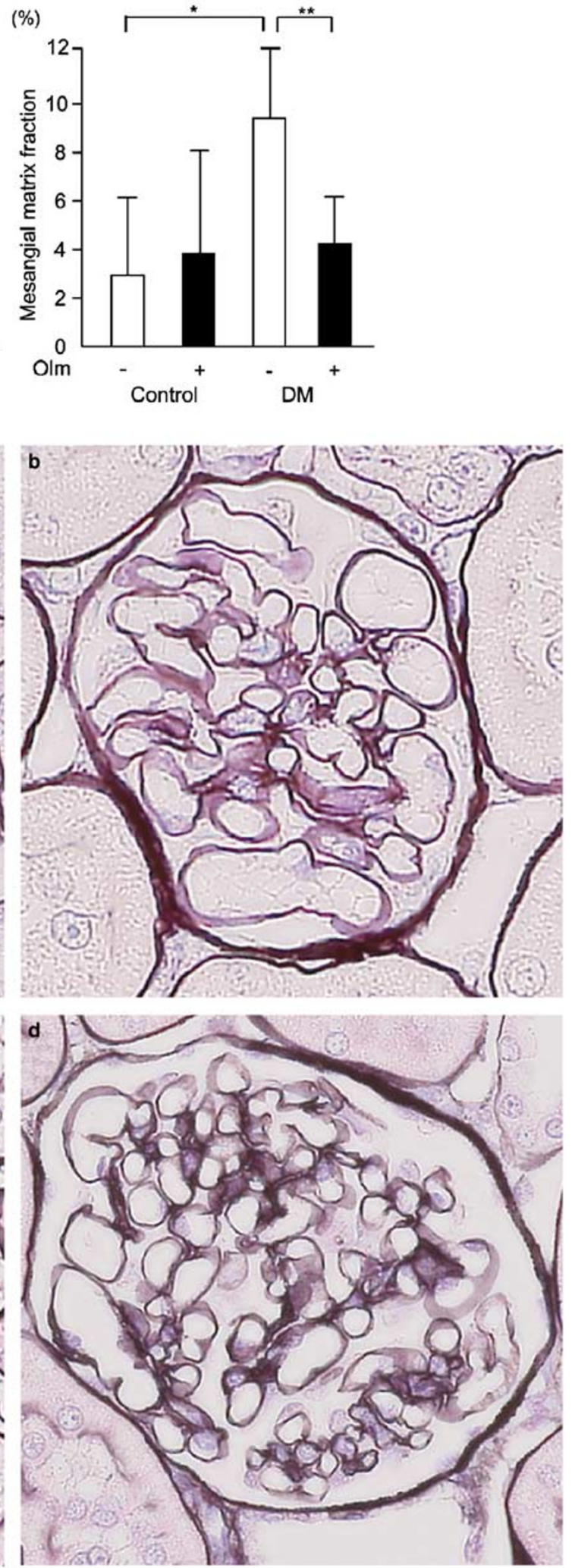

Figure 2 Mesangial matrix fraction in four groups. (A) Glomerular surface area and PASM-positive area were determined as described in Materials and methods. The mesangial sclerotic fraction was determined as percentage of mesangial matrix area per total glomerular surface area. The mesangial matrix area (left) and matrix fraction (right) in four groups were shown. ${ }^{*} P<0.05$ vs control rats treated with vehicle (group 1). ${ }^{* *} P<0.05$ vs diabetic rats treated with vehicle (group 3 ). ${ }^{* *} P<0.05$ vs control rats treated with olmesartan (group 2 ). (B) Representative light microscopic pictures of glomeruli (PASM staining, $\times 400$ original magnification) from control rats treated with vehicle (group 1) (a), control rats treated with olmesartan (group 2) (b), diabetic rats treated with vehicle (group 3) (c), and diabetic rats treated with olmesartan (group 4) (d). 

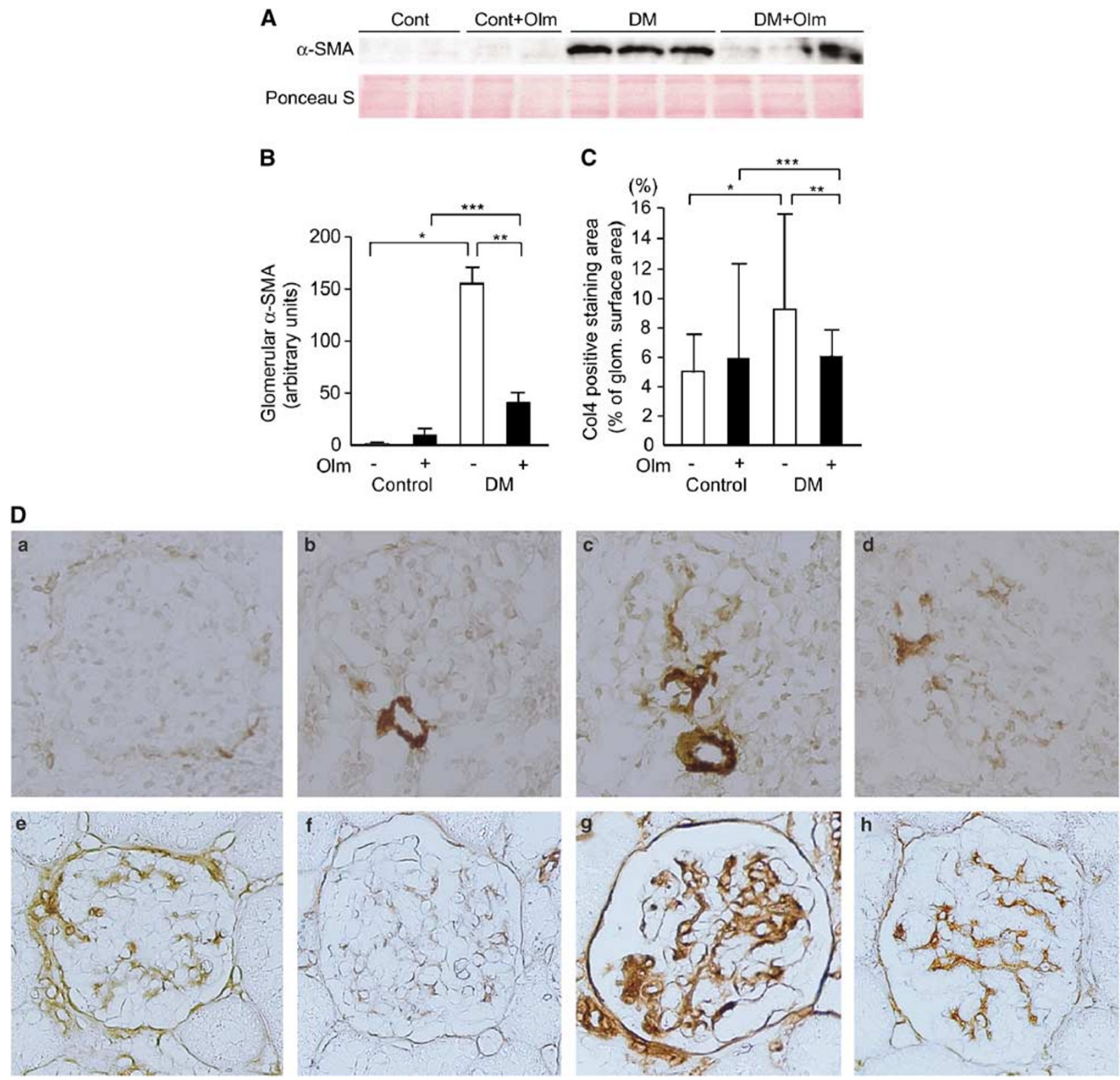

Figure 3 Glomerular expression of $\alpha$-SMA and Col4 in four groups. (A) Immunoblots for $\alpha$-SMA in the four groups are shown. Protein $(30 \mu \mathrm{g})$ obtained from each glomerular lysates was loaded. Each lane represents a representative Western blotting for the glomerular lysates from each rat. (B) Optical densitometry of glomerular expression of $\alpha$-SMA in Western blotting. ${ }^{*} P<0.05$ vs control rats treated with vehicle (group 1). ${ }^{*} P<0.05$ vs diabetic rats treated with vehicle (group 3). ${ }^{* * *} P<0.05$ vs control rats treated with olmesartan (group 2). (C) Morphometric analysis of the glomerular expression of Col4. The glomerular staining area of Col4 was measured as described in Materials and methods. ${ }^{*} P<0.05$ vs control rats treated with vehicle (group 1). ${ }^{*} P<0.05$ vs diabetic rats treated with vehicle (group 3). ${ }^{* * *} P<0.05$ vs control rats treated with olmesartan (group 2). (D) Immunohistochemistry of $\alpha$-SMA and Col4 from glomeruli of control rats with vehicle (group 1) (a, e), control rats treated with olmesartan (group 2) (b, f), diabetic rats treated with vehicle (group 3) (c, g), and diabetic rats treated with olmesartan (group 4) (d, h). Representative light microscopic appearance of glomerulus is shown. The original magnification was $\times 400$. a-d, $\alpha$-SMA; $\mathbf{e}-\mathbf{h}$, Col4.

inhibitor, can affect Ang II-induced phosphorylation of Src and Smad1. As shown in Figure 6d, PP2 inhibited the phosphorylation of Src along with Smad1. We also examined the effect of a MEK inhibitor, U0126 on Ang II-induced phosphorylation of p44/42 MAP kinase. In contrast to the effect of PP2, U0126 did not affect the phosphorylation of Smad1 (Figure 6e).

\section{Dominant Negative Src and siRNA Against Src Inhibit Ang II-Mediated Phosphorylation of Smad1 and Synthesis of Col4 in Mesangial Cells}

To elucidate the interaction between Src and Smad1, we used dominant negative Src to block the activation of Src in mesangial cells. Transfection of dominant negative Src decreased the expression of 


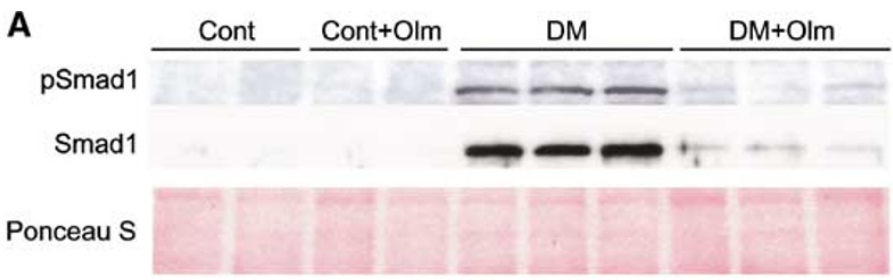

B
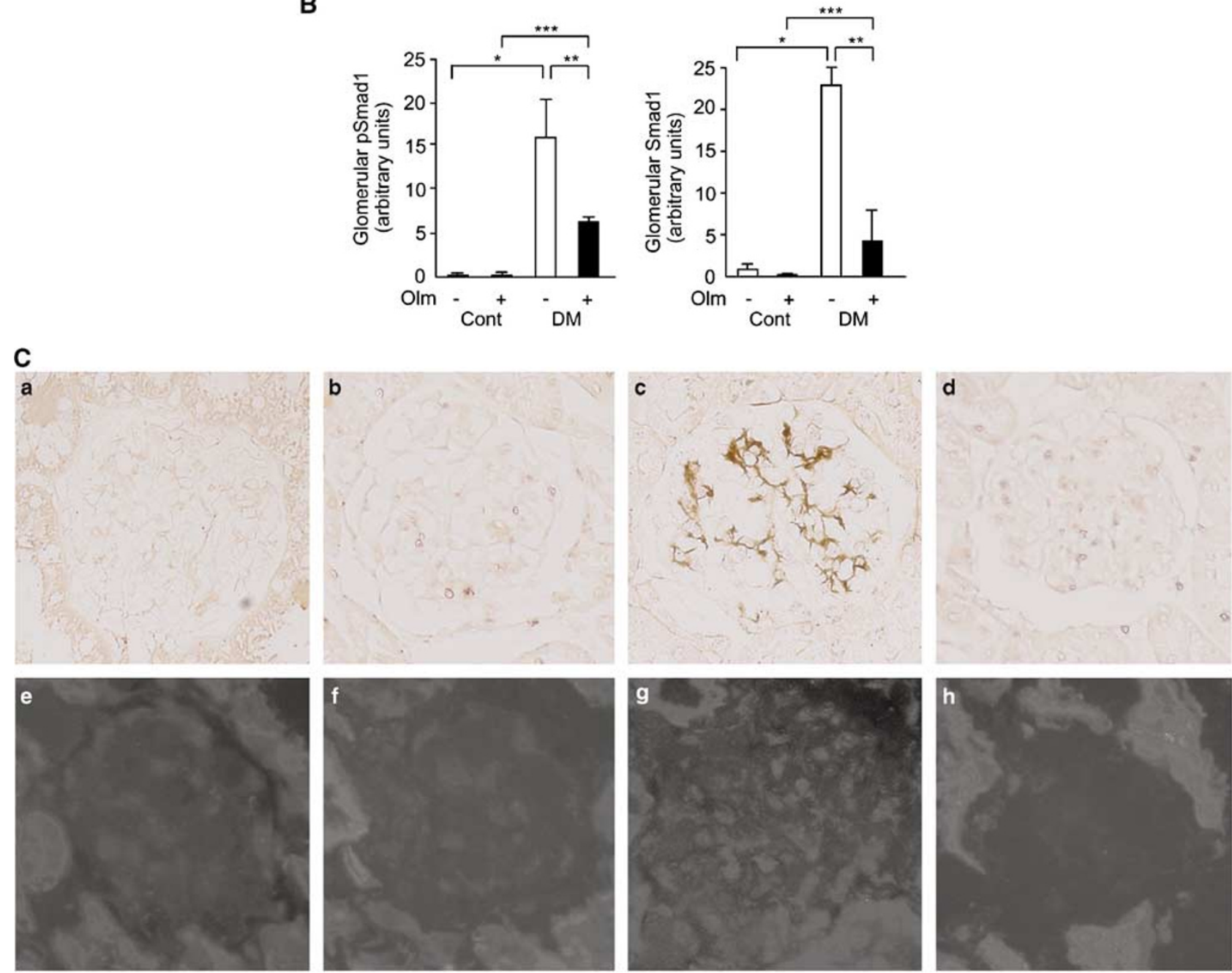

Figure 4 Glomerular expression of Smad1 and phospho-Smad1 (pSmad1) in four groups. (A) Immunoblots for Smad1 and pSmad1 in the four groups are shown. Glomerular lysates were prepared as described in Figure 3. In total, $30 \mu \mathrm{g}$ of each sample was analyzed. Each lane represents a representative Western blotting for the glomerular lysates from each rat. (B) Optical densitometry of glomerular expression of Smad1 and pSmad1 in Western blotting. ${ }^{*} P<0.05$ vs control rats treated with vehicle (group 1 ). ${ }^{*} P<0.05$ vs diabetic rats treated with vehicle (group 3). ${ }^{* *} P<0.05$ vs control rats treated with olmesartan (group 2). (C) Immunohistochemistry of Smad1 and pSmad1 in control rats treated with vehicle (group 1) (a, e), control rats treated with olmesartan (group 2) (b, f), diabetic rats treated with vehicle (group 3) (c, g), and diabetic rats treated with olmesartan (group 4) (d, h). Representative light microscopic appearance of glomerulus is shown. The original magnification was $\times 400$. a-d, Smad1; $\mathbf{e}-\mathbf{h}$, phospho-Smad1.

pSmad1 and Col4 under Ang II stimulation compared with mock vector. In contrast, $\beta$-actin protein levels, used as an internal control, were invariant across the samples (Figure 7). Next, mesangial cells were transfected with siRNA against Src or an equimolar amount of negative control siRNA and then treated with Ang II. Addition of siRNA against Src suppressed Ang II-induced phosphorylation of Src and Smad1, and synthesis of Col4. In contrast, $\beta$ - actin protein levels, used as an internal control, were invariant across the samples (Figure 8).

\section{Discussion}

In this study, we demonstrate that the AT1 antagonist ameliorates mesangial matrix expansion in the early phase of diabetic nephropathy through 
A

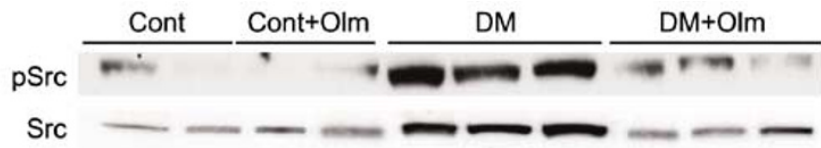

Ponceau S

B
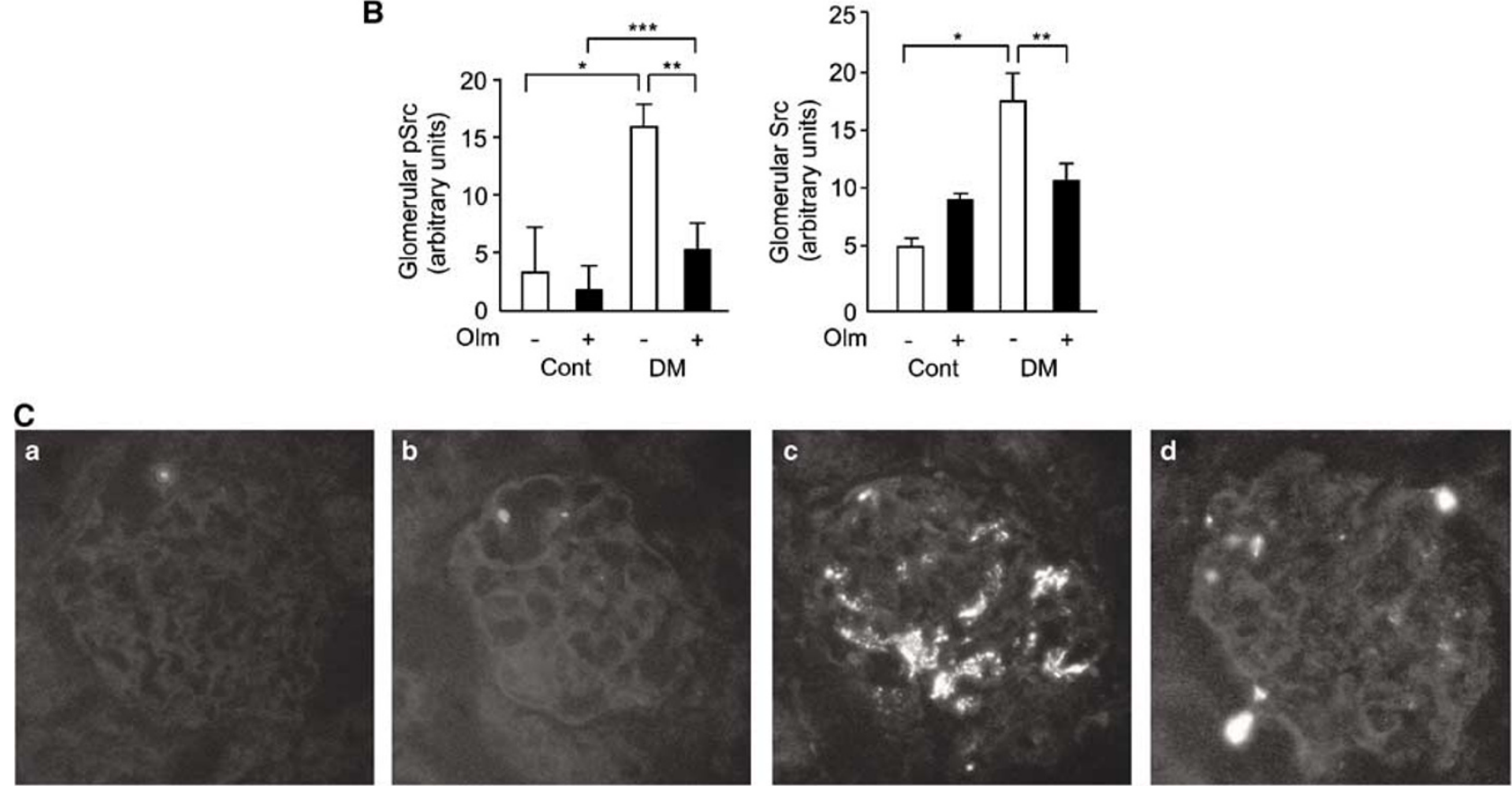

Figure 5 Glomerular expressions of Src and phospho-Src (pSrc) in four groups. (A) Immunoblots for Src and pSrc in the four groups are shown. Glomerular lysates were prepared as described in Figure 3. In all, $30 \mu \mathrm{g}$ of each sample was analyzed. Each lane represents a representative Western blotting for the glomerular lysates from each rat. (B) Optical densitometry of glomerular expressions of Src and pSrc in Western blotting. ${ }^{*} P<0.05$ vs control rats treated with vehicle (group 1 ). ${ }^{*} P<0.05$ vs diabetic rats treated with vehicle (group 3 ). ${ }^{* *} P<0.05$ vs control rats treated with olmesartan (group 2). (C) Immunohistochemistry of pSrc in control rats treated with vehicle (group 1) (a), control rats treated with olmesartan (group 2) (b), diabetic rats treated with vehicle (group 3) (c), and diabetic rats treated with olmesartan (group 4) (d). Representative light microscopic appearance of glomerulus is shown. The original magnification was $\times 400$.
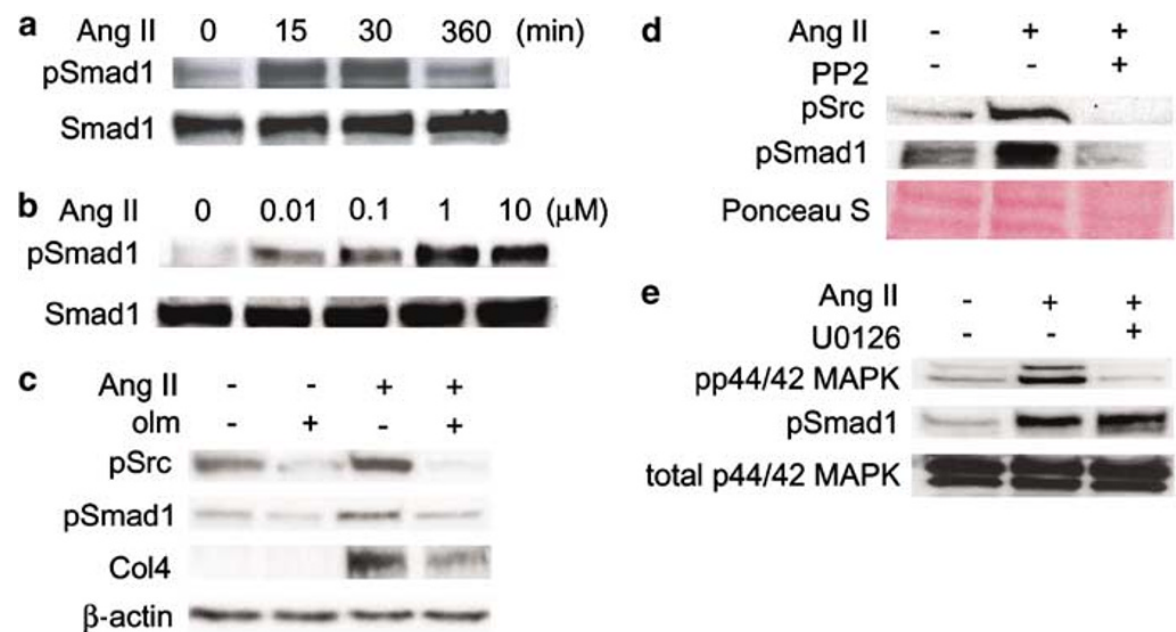

Figure 6 Role of Smad1 and Src activation by Ang II in mesangial cells. (a) Time course of phosphorylation of Smad1 by Ang II. Serumdeprived mesangial cells were treated with $0.1 \mu \mathrm{M}$ Ang II for the indicated times. (b) Dose response of phosphorylation of Smad1 by Ang II. Cells were treated with various concentrations of Ang II (0-10 $\mu \mathrm{M})$ for $15 \mathrm{~min}$. (c) Effect of olmesartan on pSmad1, pSrc, and Col4. Mesangial cells were preincubated with olmesartan ( $10 \mu \mathrm{M})$ for $48 \mathrm{~h}$ before exposure to Ang II ( $0.1 \mu \mathrm{M}, 15$ min). One of three independent experiments is shown. (d) Effect of PP2 on pSmad1 and pSrc. Mesangial cells were preincubated with PP2 $(10 \mu \mathrm{M})$ for $48 \mathrm{~h}$ before exposure to Ang II (0.1 $\mathrm{M}, 15 \mathrm{~min})$. A representative data from three independent experiments is shown. (e) Effect of U0126 on pSmad1 and phospho-p44/42 MAP kinase (pp44/42 MAP kinase). Mesangial cells were preincubated with U0126 (10 $\mu \mathrm{M}$ ) for $48 \mathrm{~h} \mathrm{before} \mathrm{exposure}$ to Ang II (0.1 $\mu \mathrm{M}, 15 \mathrm{~min})$. A representative data from three independent experiments is shown. 


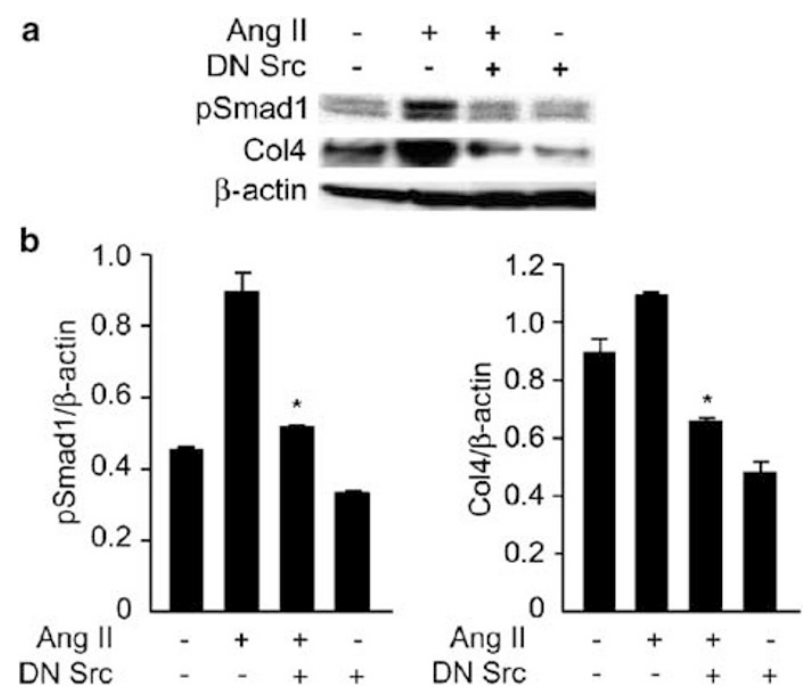

Figure 7 Effects of dominant negative Src on Ang II-mediated signaling in mesangial cells. (a) Effect of dominant negative Src on pSmad1 and Col4 in mesangial cells. After mesangial cells were transfected with dominant negative Src or mock vector, cells were starved and stimulated with Ang II $(0.1 \mu \mathrm{M}, 15 \mathrm{~min})$. A representative data from three independent experiments is shown. (b) Optical densitometry of pSmad1 and Col4 in Western blotting. ${ }^{*} P<0.05$ vs mock vector with Ang II stimulation.

the inhibition of the Smad1- and Src-mediated pathway. We also demonstrated that Ang II could stimulate Col4 production and induce a mesangial phenotypic change through Src and Smad1. This is the first demonstration showing the molecular mechanism of Ang II and AT1 through the Src/ Smad1 pathway on mesangial matrix expansion in diabetic nephropathy.

In mesangial matrix expansion, mesangial cells undergo a phenotypic change with markedly upregulated expression of Col4 and $\alpha$-SMA. ${ }^{34,37}$ It is well known that Ang II plays an important role in the development of mesangial matrix expansion. ${ }^{14,38}$ Previous studies have reported that transforming growth factor- $\beta$ (TGF- $\beta$ ) and its downstream signaling as a critical factor in mediating mesangial matrix expansion in diabetes and that one mechanism whereby Ang II promotes diabetic mesangial matrix expansion is by stimulating TGF- $\beta$ production. ${ }^{39,40}$ However, TGF- $\beta$ is not sufficient for the induction of mesangial matrix expansion. ${ }^{41}$ Therefore, another signaling pathway might present to activate Smad1, inducing mesangial matrix expansion in the early phase of diabetic nephropathy. In this regard, we propose here that Ang II can also activate Smad1 via an Src-dependent pathway in the development of mesangial matrix expansion in the early phase of diabetic nephropathy. Therefore, we tried to dissect the role of RAS in the development of mesangial matrix expansion in the early phase of diabetic nephropathy in STZ diabetic models. These models are acceptable, because they can mimic those occurred in the early phase of human diabetic nephropathy, in terms of the association between

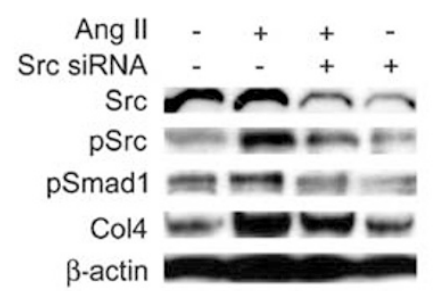

b
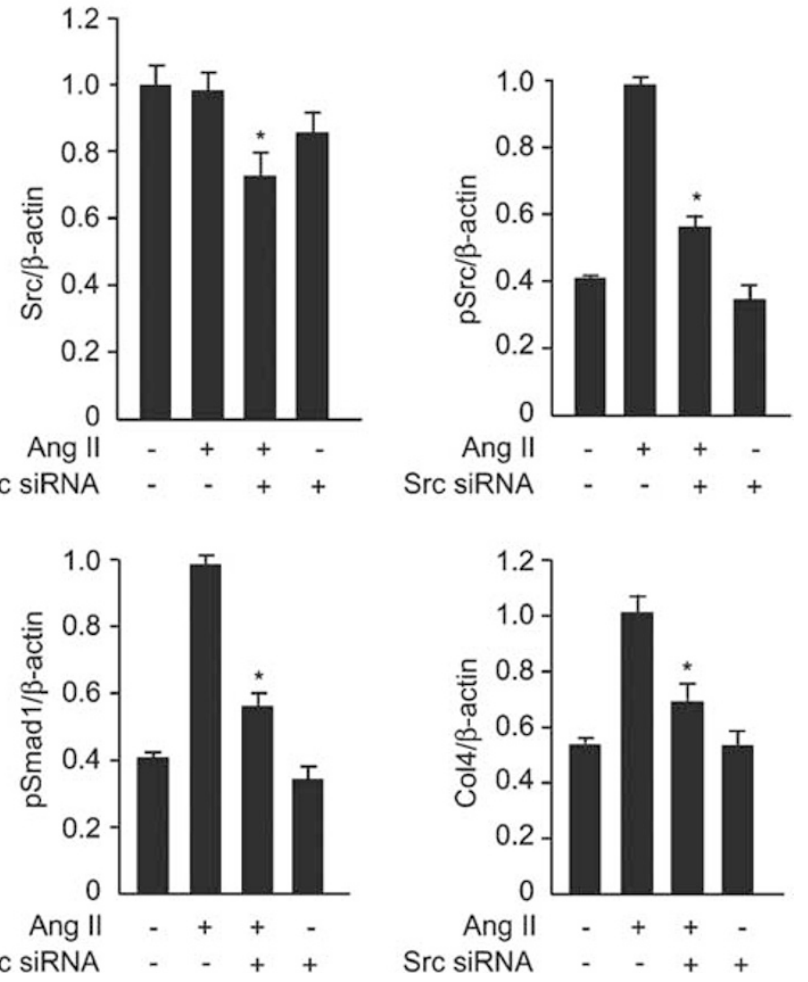

Figure 8 Effects of siRNA against Src on of Ang II-mediated signaling in mesangial cells. (a) Effect of siRNA knockdown of Src on Src, pSrc, pSmad1, and Col4 in mesangial cells. After mesangial cells were transfected with control siRNA or Srcspecific siRNA, cells were starved and stimulated with Ang II $(0.1 \mu \mathrm{M}, 15 \mathrm{~min})$. A representative data from three independent experiments is shown. (b) Optical densitometry of Src, pSrc, pSmad1, and Col4 in Western blotting. ${ }^{*} P<0.05$ vs control siRNA with Ang II stimulation.

functional and structural changes. However, they show only minimal glomerular structural changes even after 1 year of diabetes, ${ }^{42}$ and STZ itself has virulence and carciogenecity. In this study, we demonstrated that one of the mechanisms of AT1 antagonists to improve in the early phase of diabetic nephropathy is mesangial matrix expansion through the inhibitory effect on the expression of Col4 and $\alpha$ SMA possibly through the Src/Smad1 pathway in STZ rats. This conclusion is based on our following observations. Our in vitro study demonstrated that Ang II could induce rapid phosphorylation of Smad1 and Src in cultured mesangial cells and this phosphorylation was blocked by AT1 antagonists. We also showed that Ang II induced Col4 expression, which was also blocked by AT1 antagonists. Further, $\mathrm{PP}^{43}$ significantly inhibited not only Ang 
II-induced phosphorylation of Src, but also of Smad1. The experiments by dominant negative and siRNA also confirmed the presence of interaction of Src and Smad1. Treatment of STZ rats with olmesartan clearly inhibited the phosphorylation of Src and Smad1 in the glomerulus. Thus our data indicate a novel interaction of Ang II, Src, and Smad1. So far, there is one report showing the possibility of interaction between Ang II and Smad7 in diabetic mouse. ${ }^{44}$ To our knowledge, however, there is no report showing that Src is involved in diabetic nephropathy. Therefore, our study highlights the importance of Src in diabetic nephropathy. However, a direct molecular mechanism between Src and Smad1 is still unclear. Therefore, further study is needed to delineate the signaling mechanism in details.

In this study, we treated rats with a low dose of olmesartan and found no significant difference in systolic blood pressure among the four groups studied. In addition, olmesartan attenuated diabetic mesangial matrix expansion, independent of its effects on glucose metabolism. Indeed, in this study, olmesartan-treated rats showed a slight decline in creatinine clearance and albuminuria. There was a statistical difference between the groups, but are not so large. Therefore, the effect of AT1 antagonists in this study might be relatively small. Previous studies show that cyclic stretching of cultured mesangial cells that may occur in response to glomerular hypertension leads to increased synthesis of matrix proteins and TGF- $\beta .^{45,46}$

Recently, mesangial cells have been shown to produce TGF- $\beta$ when exposed to advanced glycation end products (AGEs).$^{47}$ We also showed that chronic exposure of AGEs induces the increase of Smad1 gene activation and expression, leading to Col4 overproduction via a TGF- $\beta$-dependent pathway. ${ }^{18}$ Fukami et $a l^{48}$ showed that AGEs can induce reactive oxygen species generation, which activates TGF- $\beta$-Smad 2 signaling and subsequently induces mesangial cell hypertrophy and fibronectin synthesis by autocrine production of Ang II. Others indicate that intrarenal generation of Ang II is elevated in diabetes, which subsequently leads to TGF- $\beta$ secretion. ${ }^{40,49,50}$ These studies explained the interaction between Ang II and TGF- $\beta$ in diabetic nephropathy. Therefore, it is intriguing whether these effects of Ang II in our study are mediated through TGF- $\beta$. However, the phosphorylation of Smad1 occurred very rapidly in mesangial cells. Further, TGF- $\beta$-neutralizing antibody did not affect the phosphorylation of Smad1 induced by Ang II (data not shown). Thus, our data indicate that the Ang II-Src/Smad1 pathway is independent of TGF- $\beta$ activation in mesangial cells, supported by the observation that TGF- $\beta$ is not sufficient for the induction of mesangial matrix expansion. ${ }^{41}$

Therapeutic approaches to downregulate TGF- $\beta$ signaling under diabetic conditions provide one strategy for the treatment of diabetic nephropathy. In experimental animals, the endogeneous proteoglycan decorin, a natural inhibitor of TGF- $\beta,{ }^{51,52}$ and the use of a neutralizing TGF- $\beta$ antibody ${ }^{53,54}$ have been shown to prevent the development of diabetic nephropathy. Our study indicates that the inhibition of Src may be a useful treatment for diabetic nephropathy. However, these experimental therapeutic approaches have not been practical in diabetic patients yet. Therefore, the administration of AT1 antagonists, which can downregulate the signaling of Src/Smad1 should be a first-line therapy for diabetic nephropathy.

Ang II binding to AT1 receptor triggers the activation of $\mathrm{Ca}^{2+}$ signaling and protein kinase C. The signal is then transmitted to tyrosine ( $\mathrm{Src}$ ) phosphorylation, mitogen-activated protein kinase leading to proliferation. ${ }^{55}$ In this study, we have shown the direct link between Src and Smad1 activation, and subsequent increase of Col4 synthesis in mesangial cells. However, the phosphorylation of Smad1 is independent of p44/42 MAP kinase activation in mesangial cells. Therefore, the Ang IIdependent Src-Smad1 pathway is specifically responsible for the development of diabetic mesangial matrix expansion.

In conclusion, our study indicates that Ang II can modulate Smad1-mediated signaling in diabetic mesangial matrix expansion via an Src-dependent pathway. Our study also implies that AT1 antagonists can prevent the glomerular structural changes in the early phase of diabetic nephropathy through the inhibition of Src and Smad1.

\section{Acknowledgements}

We thank Hideo Uchiyama (Taigenkai Hospital), Maki Watanabe, and Ayumi Hosotani (Kyoto University) for excellent technical assistance. This study was supported by Grants-in Aid from the Ministry of Education, Culture, Science, Sports, and Technology of Japan; Center of Excellence grants from the Ministry of Education, Culture, Science, Sports, and Technology of Japan (12CE2006); a research grant for health sciences from the Japanese Ministry of Health, Labor, and Welfare; and a grant from Takeda Medical Research Foundation.

\section{References}

1 Striker GE, Schainuck LI, Cutler RE, et al. Structuralfunctional correlations in renal disease. I. A method for assaying and classifying histopathologic changes in renal disease. Hum Pathol 1970;1:615-630.

2 Alpers CE, Hudkins KL, Gown AM, et al. Enhanced expression of 'muscle-specific' actin in glomerulonephritis. Kidney Int 1992;41:1134-1142.

3 Ziyadeh FN. The extracellular matrix in diabetic nephropathy. Am J Kidney Dis 1993;22:736-744.

4 Fogo AB. Diabetic nephropathy: it's in the numbers. Kidney Int 2002;61:2274-2275. 
5 Lewis EJ, Hunsicker LG, Bain RP, et al. The effect of angiotensin-converting-enzyme inhibition on diabetic nephropathy. The Collaborative Study Group. N Engl J Med 1993;329:1456-1462.

6 Brenner BM, Cooper ME, de Zeeuw D, et al. Effects of losartan on renal and cardiovascular outcomes in patients with type 2 diabetes and nephropathy. N Engl J Med 2001;345:861-869.

7 Lewis EJ, Hunsicker LG, Clarke WR, et al. Renoprotective effect of the angiotensin-receptor antagonist irbesartan in patients with nephropathy due to type 2 diabetes. N Engl J Med 2001;345:851-860.

8 Ruggenenti P, Perna A, Gherardi G, et al. Renal function and requirement for dialysis in chronic nephropathy patients on long-term ramipril: REIN follow-up trial. Gruppo Italiano di Studi Epidemiologici in Nefrologia (GISEN). Ramipril Efficacy in Nephropathy. Lancet 1998;352:1252-1256.

9 Mimran A, Alfaro V. Candesartan: nephroprotective effects and treatment of diabetic nephropathy. Drugs Today (Barc) 2003;39:439-450.

10 Anderson S, Jung FF, Ingelfinger JR. Renal reninangiotensin system in diabetes: functional, immunohistochemical, and molecular biological correlations. Am J Physiol 1993;265:F477-F486.

11 Anderson S. Role of local and systemic angiotensin in diabetic renal disease. Kidney Int Suppl 1997;63: S107-S110.

12 Ichikawa I, Brenner BM. Glomerular actions of angiotensin II. Am J Med 1984;76:43-49.

13 Anderson S, Rennke HG, Brenner BM. Therapeutic advantage of converting enzyme inhibitors in arresting progressive renal disease associated with systemic hypertension in the rat. J Clin Invest 1986;77:19932000.

14 Fogo AB. The role of angiotensin II and plasminogen activator inhibitor-1 in progressive glomerulosclerosis. Am J Kidney Dis 2000;35:179-188.

15 Striker LJ, Doi T, Elliot S, et al. The contribution of glomerular mesangial cells to progressive glomerulosclerosis. Semin Nephrol 1989;9:318-328.

16 Striker LJ, Peten EP, Elliot SJ, et al. Mesangial cell turnover: effect of heparin and peptide growth factors. Lab Invest 1991;64:446-456.

17 Stokes MB, Hudkins KL, Zaharia V, et al. Up-regulation of extracellular matrix proteoglycans and collagen type I in human crescentic glomerulonephritis. Kidney Int 2001;59:532-542.

18 Abe H, Matsubara T, Iehara N, et al. Type IV collagen is transcriptionally regulated by Smad 1 under advanced glycation end product (AGE) stimulation. J Biol Chem 2004;279:14201-14206.

19 Matsubara T, Abe H, Arai H, et al. Expression of Smad1 is directly associated with mesangial matrix expansion in rat diabetic nephropathy. Lab Invest 2006;86: 357-368.

20 Brown MT, Cooper JA. Regulation, substrates and functions of src. Biochim Biophys Acta 1996;1287: 121-149.

21 Fessart D, Simaan M, Laporte SA. c-Src regulates clathrin adapter protein 2 interaction with beta-arrestin and the angiotensin II type 1 receptor during clathrin-mediated internalization. Mol Endocrinol 2005;19:491-503.

22 Waters CM, Connell MC, Pyne S, et al. c-Src is involved in regulating signal transmission from
PDGFbeta receptor-GPCR(s) complexes in mammalian cells. Cell Signal 2005;17:263-277.

23 Kansra S, Stoll SW, Johnson JL, et al. Src family kinase inhibitors block amphiregulin-mediated autocrine ErbB signaling in normal human keratinocytes. Mol Pharmacol 2005;67:1145-1157.

24 Liu J, Huang C, Zhan X. Src is required for cell migration and shape changes induced by fibroblast growth factor 1. Oncogene 1999;18:6700-6706.

25 Taneda S, Hudkins KL, Topouzis S, et al. Obstructive uropathy in mice and humans: potential role for PDGF$\mathrm{D}$ in the progression of tubulointerstitial injury. J Am Soc Nephrol 2003;14:2544-2555.

26 Hudkins KL, Gilbertson DG, Carling M, et al. Exogenous PDGF-D is a potent mesangial cell mitogen and causes a severe mesangial proliferative glomerulopathy. J Am Soc Nephrol 2004;15:286-298.

27 Takahashi T, Abe H, Arai H, et al. Activation of STAT3/ Smad1 is a key signaling pathway for progression to glomerulosclerosis in experimental glomerulonephritis. J Biol Chem 2005;280:7100-7106.

28 Flyvbjerg A, Bennett WF, Rasch R, et al. Inhibitory effect of a growth hormone receptor antagonist (G120K-PEG) on renal enlargement, glomerular hypertrophy, and urinary albumin excretion in experimental diabetes in mice. Diabetes 1999;48:377-382.

29 Nangaku M, Miyata T, Sada T, et al. Anti-hypertensive agents inhibit in vivo the formation of advanced glycation end products and improve renal damage in a type 2 diabetic nephropathy rat model. J Am Soc Nephrol 2003;14:1212-1222.

30 Makibayashi K, Tatematsu M, Hirata M, et al. A vitamin $\mathrm{D}$ analog ameliorates glomerular injury on rat glomerulonephritis. Am J Pathol 2001;158:17331741.

31 Yamamoto Y, Kato I, Doi T, et al. Development and prevention of advanced diabetic nephropathy in RAGEoverexpressing mice. J Clin Invest 2001;108:261-268.

32 Nagai K, Matsubara T, Mima A, et al. Gas6 induces Akt/mTOR-mediated mesangial hypertrophy in diabetic nephropathy. Kidney Int 2005;68:552-561.

33 Pippin JW, Qu Q, Meijer L, et al. Direct in vivo inhibition of the nuclear cell cycle cascade in experimental mesangial proliferative glomerulonephritis with Roscovitine, a novel cyclin-dependent kinase antagonist. J Clin Invest 1997;100:2512-2520.

34 Takeoka $\mathrm{H}$, Iehara $\mathrm{N}$, Uematsu-Yanagita $\mathrm{M}$, et al. A multifunctional transcription factor (A1p145) regulates the smooth muscle phenotype in mesangial cells. Biochem Biophys Res Commun 1998;252: 290-295.

35 Nagai K, Arai H, Yanagita M, et al. Growth arrestspecific gene 6 is involved in glomerular hypertrophy in the early stage of diabetic nephropathy. J Biol Chem 2003;278:18229-18234.

36 Stockelman MG, Lorenz JN, Smith FN, et al. Chronic renal failure in a mouse model of human adenine phosphoribosyltransferase deficiency. Am J Physiol 1998;275:F154-F163.

37 Abe H, Iehara N, Utsunomiya $\mathrm{K}$, et al. A vitamin D analog regulates mesangial cell smooth muscle phenotypes in a transforming growth factor-beta type II receptor-mediated manner. J Biol Chem 1999;274: 20874-20878.

38 Fogo AB. Mechanisms in nephrosclerosis and hypertension-beyond hemodynamics. J Nephrol 2001; 14(Suppl 4):S63-S69. 
39 Border WA, Noble NA. Interactions of transforming growth factor-beta and angiotensin II in renal fibrosis. Hypertension 1998;31:181-188.

40 Weigert C, Brodbeck K, Klopfer K, et al. Angiotensin II induces human TGF-beta 1 promoter activation: similarity to hyperglycaemia. Diabetologia 2002;45: 890-898.

41 Yang CW, Striker GE, Chen WY, et al. Differential expression of glomerular extracellular matrix and growth factor mRNA in rapid and slowly progressive glomerulosclerosis: studies in mice transgenic for native or mutated growth hormone. Lab Invest 1997;76:467-476.

42 Zatz R, Meyer TW, Rennke HG, et al. Predominance of hemodynamic rather than metabolic factors in the pathogenesis of diabetic glomerulopathy. Proc Natl Acad Sci USA 1985;82:5963-5967.

43 Hanke JH, Gardner JP, Dow RL, et al. Discovery of a novel, potent, and Src family-selective tyrosine kinase inhibitor. Study of Lck- and FynT-dependent T cell activation. J Biol Chem 1996;271:695-701.

44 Liao J, Kobayashi M, Kanamuru Y, et al. Effects of candesartan, an angiotensin II type 1 receptor blocker, on diabetic nephropathy in KK/Ta mice. J Nephrol 2003;16:841-849.

45 Riser BL, Cortes $\mathrm{P}$, Zhao X, et al. Intraglomerular pressure and mesangial stretching stimulate extracellular matrix formation in the rat. J Clin Invest 1992;90:1932-1943.

46 Riser BL, Cortes P, Heilig C, et al. Cyclic stretching force selectively up-regulates transforming growth factor-beta isoforms in cultured rat mesangial cells. Am J Pathol 1996;148:1915-1923.

47 Throckmorton DC, Brogden AP, Min B, et al. PDGF and TGF-beta mediate collagen production by mesangial cells exposed to advanced glycosylation end products. Kidney Int 1995;48:111-117.

48 Fukami K, Ueda S, Yamagishi S, et al. AGEs activate mesangial TGF-beta-Smad signaling via an angiotensin II type I receptor interaction. Kidney Int 2004;66: 2137-2147.

49 Nagai Y, Yao L, Kobori H, et al. Temporary angiotensin II blockade at the prediabetic stage attenuates the development of renal injury in type 2 diabetic rats. J Am Soc Nephrol 2005;16:703-711.

50 Singh R, Alavi N, Singh AK, et al. Role of angiotensin II in glucose-induced inhibition of mesangial matrix degradation. Diabetes 1999;48:2066-2073.

51 Border WA. Transforming growth factor-beta and the pathogenesis of glomerular diseases. Curr Opin Nephrol Hypertens 1994;3:54-58.

52 Isaka Y, Brees DK, Ikegaya K, et al. Gene therapy by skeletal muscle expression of decorin prevents fibrotic disease in rat kidney. Nat Med 1996;2: 418-423.

53 Ziyadeh FN, Hoffman BB, Han DC, et al. Long-term prevention of renal insufficiency, excess matrix gene expression, and glomerular mesangial matrix expansion by treatment with monoclonal antitransforming growth factor-beta antibody in $\mathrm{db} / \mathrm{db}$ diabetic mice. Proc Natl Acad Sci USA 2000;97: 8015-8020.

54 Chen S, Iglesias-de la Cruz MC, Jim B, et al. Reversibility of established diabetic glomerulopathy by anti-TGF-beta antibodies in $\mathrm{db} / \mathrm{db}$ mice. Biochem Biophys Res Commun 2003;300:16-22.

55 Sabri A, Govindarajan G, Griffin TM, et al. Calciumand protein kinase C-dependent activation of the tyrosine kinase PYK2 by angiotensin II in vascular smooth muscle. Circ Res 1998;83:841-851. 